\title{
FIRST ASSESSMENT OF POLLUTION IMPACT AT ESSAOUIRA COAST (MOROCCO) USING BIOTIC AND ABIOTIC PARAMETERS AND THE RED ALGAE ELLISOLANDIA ELONGATA AS POTENTIAL BIOINDICATOR OF ORGANIC POLLUTION
}

SABRI, H. ${ }^{1,2^{*}}-$ BOULARHBAR, H. $.^{1,2}-$ MAAROUF, A. ${ }^{3}-$ SBAHI, S. ${ }^{1,2}-$ HASNI, M. $^{4}-$ BOUNDIR, Y. ${ }^{1,2}-$ MANDI, L. ${ }^{1,2}-$ KERNER, M. ${ }^{5}-$ WEINBERGER, F. ${ }^{6}-$ CHERIFI, O. ${ }^{1,2}$

${ }^{1}$ Laboratory of Water, Biodiversity and Climate Change, Faculty of Sciences Semlalia, Cadi Ayyad University, Bd. Prince My Abdellah, PO Box 2390, 40000 Marrakech, Morocco

${ }^{2}$ National Center for Studies and Research on Water and Energy (CNEREE), Cadi Ayyad University, Avenue Abdelkrim Khattabi, PO Box 511, 40000 Marrakech, Morocco

${ }^{3}$ Laboratory of Food, Environment and Health (LAES), Faculty of Sciences and Technics, Cadi Ayyad University, Avenue Abdelkrim Khattabi, PO Box 549, 40000 Marrakech, Morocco

${ }^{4}$ Faculty of Sciences, Ibn Zohr University, PO Box 8106, 80000 Agadir, Morocco

${ }^{5}$ SSC Strategic Science Consult, Hamburg, Germany

${ }^{6}$ GEOMAR Helmholtz-Center for Ocean Research, Kiel, Germany

*Corresponding author

e-mail: hasnae.sabri@ced.uca.ma; phone: +21-263-842-2749; fax: +21-205-433-170

(Received $30^{\text {th }}$ Jun 2020; accepted $17^{\text {th }}$ Sep 2020)

\begin{abstract}
Environmental pollutants might significantly affect the ecological integrity of coastal waters. Biological indicators like seaweeds have been used globally to assess water pollution. In the present work, the seaweed Ellisolandia elongata was used to evaluate the pollution status in coastal waters around Essaouira city. Three sites were chosen: One as reference station (S1) and two polluted ones (S2 and S3). Seaweed biodiversity, physiologic parameters of E. elongata, as well as, abiotic parameters were studied. Results showed that at the polluted stations, seaweed biodiversity was significantly lower than in S1. However, the concentrations of Total Suspended Solids, Electrical Conductivity, Biological Oxygen Demand, Chemical Oxygen Demand, Ammonium, and Orthophosphates at S2 were significantly higher than at S1. Metal content of E. elongata remained below detection limit at all stations except for $\mathrm{Zn}$ and $\mathrm{Cu}$ at S2 considered the most polluted station. With respect to the physiologic parameters, Proline, Glycine Betaine and Polyphenol contents at S2 were above, whereas, Chlorophyll a content and axis length were below those determined at the reference station. From the results it can be concluded that seaweed E. elongata could be a good indicator to determine organic pollution in marine ecosystems.

Keywords: seaweed, organic pollution, physicochemical parameters, Ellisolandia elongata physiology, biomonitoring
\end{abstract}

\section{Introduction}

Boarding to the Atlantic Ocean and the Mediterranean Sea, the Moroccan coastlines are exposed to various types of anthropogenic stress. This environment is constantly exposed to human influence through domestic and industrial waste waters, which often introduce high quantities of pollutants without pre-treatment. In addition, the habitats undergo physical destruction which produces a significant impact on the coastal 
environment and lead to significant ecological deterioration (Islam and Tanaka, 2004; Moore et al., 2004; Gu et al., 2007; Amin et al., 2009; Er-Raioui et al., 2012; Hong et al., 2020), causing serious changes in marine organisms, including macrofauna, seagrasses, algae and others (Sivadas et al., 2010; Sabri et al., 2017; Boundir et al., 2019). In fact, algae are the basis of many marine food webs. Their composition fluctuates depending on several conditions, such as temperature, light, salinity, $\mathrm{pH}$, and nutrients (Huertas et al., 2011). According to literature, it is known that the amount of macronutrient of coastal areas are the main factors which control the structural composition of algae communities (McGlathery et al., 2007; Leterme et al., 2014; Nassar et al., 2015). Celis-Plá et al. (2014) demonstrated that Nitrate, ammonium and orthophosphate are essential macronutrients for algae growth. However, these nutrients can have a serious negative impact on algal communities if they reach high concentrations near urban areas (Guerra-García and Koonjul, 2005; Nassar et al., 2015). In addition, urbanization and industrialization which increases due to population expansion is often coupled to a release of heavy metals into coastal waters which are considered among the most serious contaminants of aquatic ecosystems due to their high potential to enter and accumulate in food chains (Erdoğrul and Erbilir, 2007).

However, only limited published information on organic and inorganic pollutants is available for the Moroccan coast, and in particular for its Atlantic section (Kaimoussi et al., 2002; Mouradi et al., 2014; Rezzoum et al., 2016). At certain concentrations, these compounds could have an impact on seaweeds, causing disappearance of some species or declining or shifting biodiversity (DÍez et al., 1999; Nassar et al., 2015; El-adl et al., 2017; Sabri et al., 2017). Many authors used seaweeds to evaluate the degree of pollution, as they are considered to be excellent indicators of environmental change (Harley et al., 2006; De Faveri et al., 2010; Benkdad et al., 2011; Reis et al., 2014; Shams El-Din et al., 2014; García-Seoane et al., 2018). When seaweeds are exposed to stress (e.g. heavy metals or pathogens) they may respond in characteristic ways, such as decrease of axis length or chlorophyll content. Also overproduction of specific metabolic products, such as proline, glycine betaine (GB) or total phenolic compounds (TPC) may compensate the cellular imbalances caused by environmental stress (Häder et al., 1997; Coelho et al., 2000; Di Martino et al., 2003; Koivikko et al., 2005; Liang et al., 2013). Thus, these metabolites allow certain seaweeds to acclimatize to some degree and to ensure survival (Fatma et al., 2007; Lamalakshmi et al., 2017). Previous studies already reported that algal size and the content of molecules like chlorophyll, TPC, GB and proline may be used as bioindicators to monitor air pollution (Anbazhagan et al., 1988; Agbaire, 2016; Khairallah et al., 2018; Mukherjee et al., 2019), salt stress (Ali et al., 1999; Carillo et al., 2008; Amirjani, 2010; Fariduddin et al., 2013), drought stress (Si et al., 2015; Mirshad and Puthur, 2016; Mao et al., 2019) and freezing stress (Nomura et al., 1995), as well as inorganic (Alia and Saradhi, 1991; Amna et al., 2015; Varun et al., 2015; Saif and Khan, 2018; Boundir et al., 2019) and organic pollution (Abdel and Abdel, 2015; Bibi et al., 2019).

A precondition to use seaweeds as a bioindicator is that they are present in the areas under study. This is the case for the Corallinaceae which exist in almost every habitat type, even in polluted areas, within the photic (Adey and Macintyre, 1973; Steneck, 1986; DÍez et al., 1999). Likewise, Ellisolandia elongata which occurs along the Atlantic intertidal zone of Essaouira and its surroundings was chosen in the present study to investigate its applicability as a bioindicator. For doing so, the species and numbers of macroalgae present were determined at two polluted sites and compared to 
those at an unpolluted site during different seasons in 2017/2018. In addition, variations in the content of selected metabolic substances and heavy metals of the tissue of Ellisolandia elongata samples were determined and compared together with the seasonal differences of the physicochemical parameters of the waters at the study sites.

\section{Materials and methods}

\section{Study area}

The study area is located along the Atlantic Coast west of Central Morocco in the Essaouira Province, which is administered under the Marrakech-Safi Region government (Morocco). It extends longitudinally among $9^{\circ} 67^{\prime}$ and $9^{\circ} 77^{\prime}$ 'West and latitudinally among $31^{\circ} 51^{\prime}$ and $31^{\circ} 63^{\prime}$ North (Fig. 1).

The study area has a diverse and exceptional climate, shaped by its geographical location between the Sahara Desert and the Atlantic Ocean. The aridity increases from west to east. The western narrow coastal fringe around Essaouira is reached by cold currents coming from the Canary Islands, which generate a microclimate with a relatively homogeneous average temperature of about $16.7^{\circ} \mathrm{C}$ throughout the year. As a consequence, the difference between average temperatures in the hottest and the coldest month is relatively small (Mwambo, 2007; Bazairi et al., 2010). Agriculture, fishing, craft, mining, trade, tourism, energy production and some other industrial and recreational activities are the most dominant economic sectors in Essaouira city.

Three stations, located on rocky substrates along the Essaouira coast, were chosen for this study (Fig. 1).

- Moulay Bouzerktoune Station (S1), located approximately $30 \mathrm{~km}$ away from the city $\left(31^{\circ} 63^{\prime} \mathrm{N}-9^{\circ} 67^{\prime} \mathrm{W}\right)$, is considered as a reference station, affected by anthropogenic activities only during ephemeral tourism activities at the end of spring and during summer.

- Bab Doukkala Station (S2), the industrial district of Essaouira, located in the city, north of the port ( $\left.31^{\circ} 51^{\prime} \mathrm{N}-9^{\circ} 76^{\prime} \mathrm{W}\right)$, allows us to assess the impact of the discharges of Essaouira city on marine macroalgae. Indeed, this urban coast receives significant domestic and some industrial wastewater, as well as limited solid discharges that are mainly composed of household waste.

- Port Station (S3), also located within the city $\left(31^{\circ} 51^{\prime} \mathrm{N}-9^{\circ} 77^{\prime} \mathrm{W}\right)$, receives some industrial discharges from the port, as well as domestic discharges from the city districts.

\section{Sampling}

In order to study the phytobenthos biodiversity, seaweed samples were collected seasonally (once per season) during 2017 to 2018 in autumn, winter, spring and summer at low tide $(0$ to $1.5 \mathrm{~m})$ along the Essaouira coast at the three stations (S1, S2, S3), following the quadrat method (Ar Gall and Connan, 2004). 30 quadrats were provided for each bathymetric level because of the irregularity of algal distribution in the foreshore. Samples were transferred to the laboratory and stored at $4{ }^{\circ} \mathrm{C}$ in a cooler. The species of the seaweeds were identified in situ and at the laboratory using a magnifying glass and a binocular microscope.

A large quantity of the Corallinaceae Ellisolandia elongata (J. Ellis and Solander) K. R. Hind and G.W. Saunders with basionym Corallina elongata (Guiry and Guiry, 2020) 
was collected in order to study its physiology and metal uptake. The algal material sampled was carefully washed with seawater, in order to omit epiphytes, sediments and associated fauna, and was cleaned thereafter with distilled water to remove excess salt. The algal material thus prepared was lyophilized, ground to powder in a dust-free atmosphere and using metal-free equipment to avoid contamination and finally stored at $-20{ }^{\circ} \mathrm{C}$ for toxicological and physiological analysis.

In addition, water was sampled in glass bottles $(500 \mathrm{ml})$ and transported to the lab in a cooler on ice $\left(4{ }^{\circ} \mathrm{C}\right)$ to reduce adsorption, precipitation, contamination or evaporation (Rodier, 2009).

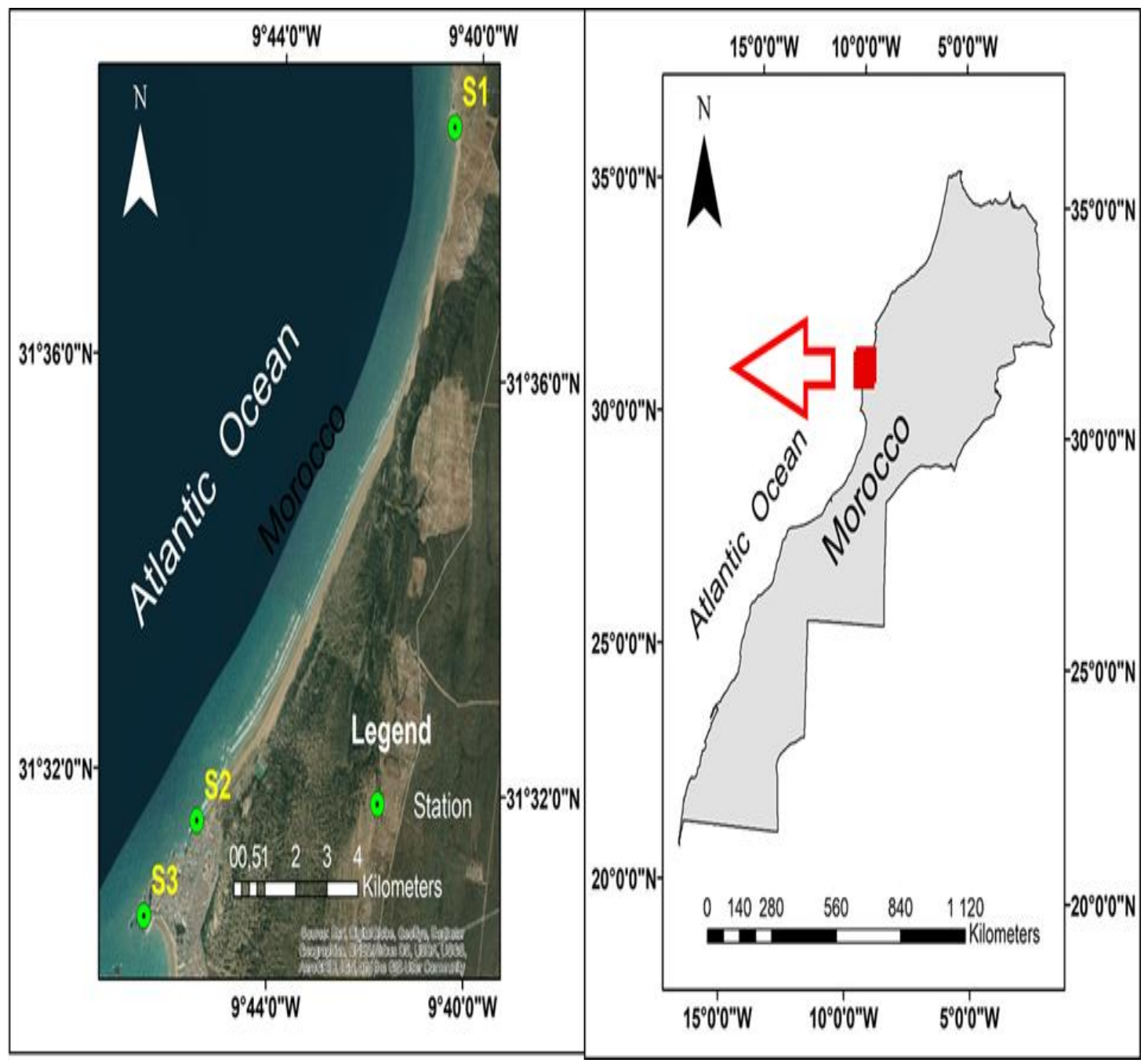

Figure 1. Geographical position of the study sites along the coast of Essaouira. (S1) My Bouzerktoune: $31^{\circ} 63^{\prime} \mathrm{N}-9^{\circ} 67^{\prime} \mathrm{W}-$ (S2) Bab Doukkala: 31 ${ }^{\circ} 51^{\prime} \mathrm{N}-9^{\circ} 76^{\prime} \mathrm{W}-(\mathrm{S} 3)$ The port: $31^{\circ} 51^{\prime} \mathrm{N}-9^{\circ} 77^{\prime} \mathrm{W}$

\section{Physicochemical parameters}

The water temperature, electrical conductivity (EC) and $\mathrm{pH}$ were determined on site with a multimeter instrument (Orion 4 Star), while dissolved oxygen (DO) was measured by a digital pocket oxymeter (HANNA-HI9829). All parameters were always measured in situ during collection of the samples at all sites and in all seasons. 
Concentrations of Total Suspended Solids (TSS) in water were determined by filtration of $200 \mathrm{ml}$ of water sample through glass filters $(0.45 \mu \mathrm{m}$ mesh $)$, which was subsequently dried and weighed. Biological Oxygen Demand (BOD5), Chemical Oxygen Demand (COD), and concentrations of Nitrogenous and Phosphorus compounds were measured according to AFNOR norms (Association Française de NORmalisation) reported in Table 1. The oxidizable matter concentrations were calculated using Equation 1:

$$
\text { Oxidizable matter }=\frac{2 \times \mathrm{BOD}_{5}+\mathrm{COD}}{3}
$$

Table 1. AFNOR norms of the studied parameters

\begin{tabular}{c|c}
\hline Parameters & AFNOR norms \\
\hline BOD $_{5}$ & NF T90-103 \\
COD & NF T90-101 \\
Total nitrogen & NF T90-061 \\
Nitrate & NF T90-012 \\
Ammonium & NF T90-015-2 \\
Total phosphorus & NF T90-023 \\
Orthophosphate & NF T90-022 \\
\hline
\end{tabular}

\section{Physiological parameters}

\section{Chlorophyll a}

Chlorophyll a (Chla), was determined according to the method of Jeffrey and Humphrey (1975), based upon extraction of $10 \mathrm{~g}$ of fresh algae in $10 \mathrm{ml}$ of acetone $90 \%$. The tubes were incubated in the dark at $4{ }^{\circ} \mathrm{C}$ for $24 \mathrm{~h}$ and subsequently centrifuged at $19,000 \mathrm{~g}$ for $30 \mathrm{~min}$. Absorbance of the resulting supernatant was measured at 662 and $644 \mathrm{~nm}$, using a spectrophotometer (Boeco Germany S20).

\section{Proline}

The algal proline content was quantified following the method of Monneveux and Nemmar (1986). $100 \mathrm{mg}$ of dried tissue was homogenized in $2 \mathrm{ml}$ of methanol (40\%) and then placed in a water bath at $85^{\circ} \mathrm{C}$ for $1 \mathrm{~h}$. After cooling the samples were centrifuged at $4000 \mathrm{~g}$ for $10 \mathrm{~min}$ at $4{ }^{\circ} \mathrm{C}$. $1 \mathrm{ml}$ of supernatant was added to a mixture composed of $1 \mathrm{ml}$ of acetic acid, $25 \mathrm{mg}$ of ninhydrin and $1 \mathrm{ml}$ of a solution composed of distilled water, glacial acetic acid and ortho-phosphoric acid of density 1.7 (120, 300, 80: v/v/v). The mixture was heated again for $30 \mathrm{~min}$ at $100{ }^{\circ} \mathrm{C}$ in a water bath and subsequently allowed to cool at room temperature, and then added to $5 \mathrm{ml}$ of toluene. The upper phase was collected and dehydrated with a pinch of anhydrous $\mathrm{Na}_{2} \mathrm{SO}_{4}$. The absorbance was read at $520 \mathrm{~nm}$.

\section{Glycine betaine (GB)}

The GB essay was performed according to the protocol of Grieve and Grattan (1983). $0.5 \mathrm{~g}$ DW of seaweed was mechanically shaken with $20 \mathrm{ml}$ of deionized water at $25{ }^{\circ} \mathrm{C}$ for $48 \mathrm{~h}$. After filtration, $0.5 \mathrm{ml}$ of extract was mixed with $0.5 \mathrm{ml}$ of $2 \mathrm{~N}$ sulfuric 
acid. Then $0.2 \mathrm{ml}$ of $\mathrm{KI}-\mathrm{I}_{2}$ reagent (containing $15.7 \mathrm{~g}$ iodine and $20 \mathrm{~g} \mathrm{KI}$ in $100 \mathrm{ml}$ ) was added and shaken in ice cold water for $1 \mathrm{~h}$. The tubes were stored at $0-4{ }^{\circ} \mathrm{C}$ for $16 \mathrm{~h}$ and then centrifuged at $10,000 \mathrm{rpm}$ for $15 \mathrm{~min}$ at $0{ }^{\circ} \mathrm{C}$. The supernatant was carefully drawn off and the pellet was dissolved in $9 \mathrm{ml}$ of 1,2-dichloroethane (chilled at $-10{ }^{\circ} \mathrm{C}$ ). The absorbance was measured after $2 \mathrm{~h}$ at $365 \mathrm{~nm}$.

\section{Total phenolic compounds (TPC)}

TPC were measured according to Silvia Taga et al. (1984). A quantity of $0.5 \mathrm{~g}$ of dried algae was extracted in 60:40 acidified methanol/water $(0.3 \% \mathrm{HC} 1)$. Supernatant at $100 \mu \mathrm{l}$ was mixed with $2 \mathrm{ml}$ of $\mathrm{Na}_{2} \mathrm{CO}_{3}(2 \%)$. After 2 min $100 \mu \mathrm{l}$ of Folin-Ciocalteu's phenol reagent $(50 \%)$ were added and the mix was allowed to stand at room temperature for $30 \mathrm{~min}$. The absorbance was then read at $750 \mathrm{~nm}$ within $2 \mathrm{~h}$. The results were expressed as gallic acid equivalents (GAE), based upon measurement of gallic acid standards ranging in concentration from $10 \mathrm{mg} \mathrm{mL}^{-1}$ to $200 \mathrm{mg} \mathrm{mL}^{-1}$.

\section{Morphometry}

The main axis length of seaweeds was determined using a non-destructive method by Murray et al. (2002), which does not require detaching the seaweed. The lengths of individual axes were measured in situ with a ruler.

\section{Heavy metals}

The method of Topcuoğlu et al. (2003) was adopted to quantify trace metals after digestion with hydrogen peroxide, sulfuric and nitric acids. If necessary, samples thus obtained were diluted depending on their metal concentrations to allow for analysis with an Atomic Absorption Spectrophotometer (AA-6300 SHIMADZU).

\section{Statistical analysis}

Morphometric measurements were conducted on 30 algal samples, while in situ measurements and laboratory analysis were established in triplicate and mean values and standard deviations were calculated. For statistical data analysis, Kruskal-Wallis and Wilcoxon tests were applied to analyze differences among the three sampling stations. Linear correlations between parameters were analyzed with the Pearson coefficient. The regression coefficient was calculated between concentrations of different compounds in wastewater, and Principal Component Analysis was used as a classification tool. The SPSS Statistics version 23 package (IBM, USA) was used for data analysis. All statistical hypothesis testing was based upon use of a probability value $\mathrm{p}$ of 0.05 .

\section{Results}

\section{Algal diversity}

A total of 309 taxa was recorded at the three sampling stations located along the coastline of Essaouira city. The maximum of specific richness was found at S1 with 221 species, followed by S3 with 140 species and S2 with only 111 species (Fig. 2). Generally, most species belonged to the Rhodophyceae (68\%), followed by the Chlorophyceae (17\%) and the Phaephyceae (16\%). The green algal species at S2 and 
S3 contributed $29 \%$ and $21 \%$, respectively, while this percentage was lower at S1 (13\%). On the other hand, the red and brown algae contributed $73 \%$ and $14 \%$, respectively, at reference station $\mathrm{S} 1$ and where thus more dominant at this station than at the polluted stations S2 and S3. The dominant species at S1 were Hypnea musciformis, Gelidium corneum, Carpodesmia tamariscifolia and Bifurcaria bifurcata. The brown seaweeds were associated with epiphytic species (e.g. Aglaothamnion tripinnatum, Polysiphonia atlantica, $P$. elongata, Lomentaria articulata, Plocamium raphelisianum, $P$. cartilagineum). The S2 and S3 stations exhibited mostly common species, which were generally dominated by the Chlorophyceae (Ulva and Codium genera), by some epiphytic species (e.g. Derbesia lamourouxii, D. tenuissima, cladophora polifera, Chaetomorpha aerea) and by the red alga Caulacanthus ustulatus.

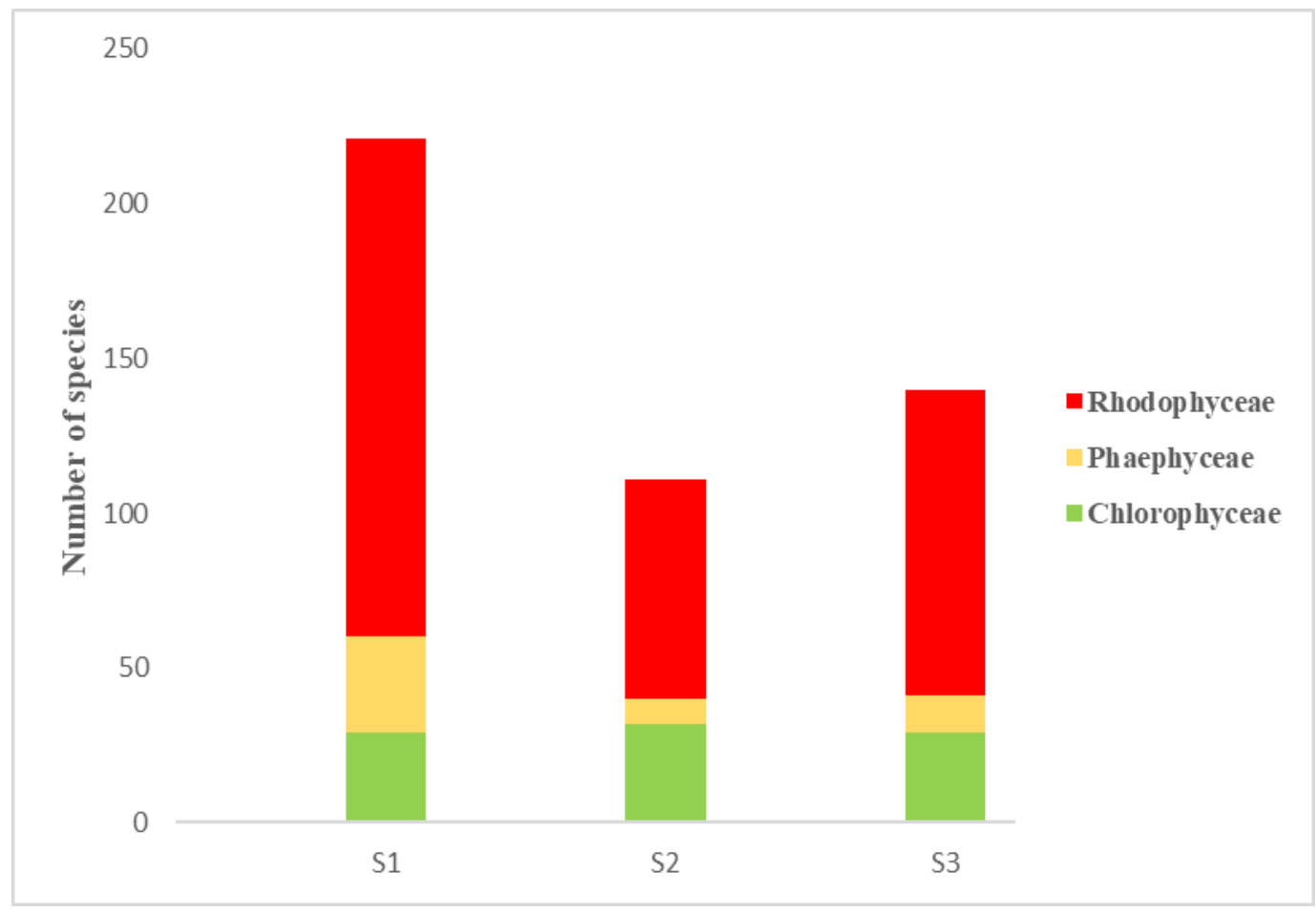

Figure 2. Algal diversity in the studied areas

\section{Physicochemical parameters}

The results of the physicochemical parameters of seawater measured in situ during the study period are presented in Table 2 .

Temperature values vary between $15.5{ }^{\circ} \mathrm{C}$ during winter and $23.46{ }^{\circ} \mathrm{C}$ during summer all recorded at $\mathrm{S} 3$. For this parameter, statistical analyses did not show clearly a significant difference between the study sites, while the other parameters showed a significant difference between the control station and the polluted ones, especially between $\mathrm{S} 1$ and $\mathrm{S} 2$ ( $\mathrm{p}<0.05)$. $\mathrm{pH}$ and DO contents ranged from 7.2 to 8.7 and from 5.6 to $8 \mathrm{mg} \mathrm{L}^{-1}$, respectively, and were significantly higher at $\mathrm{S} 1$ in comparison to the other stations (S2 and S3). In contrast, the highest values of EC were recorded at the two polluted stations. Their maxima reached $45500 \mu \mathrm{s} \mathrm{cm}^{-1}$ at S3 and $47000 \mu \mathrm{s} \mathrm{cm}^{-1}$ at S2, but only $39000 \mu \mathrm{sm}^{-1}$ at $\mathrm{S} 1$. 
Table 2. Seasonal variation of temperature $\left({ }^{\circ} \mathrm{C}\right), \mathrm{pH}$, electrical conductivity $\left(\mu \mathrm{s} \mathrm{cm}^{-1}\right)$, and dissolved oxygen $\left(m g L^{-1}\right)$ in the seawater at the three studied areas

\begin{tabular}{c|c|c|c|c|c}
\hline Season & Station & $\mathbf{T}$ & $\mathbf{p H}$ & $\mathbf{E C}$ & DO \\
\hline \multirow{4}{*}{ Autumn } & $\mathrm{S} 1$ & $18.6 \pm 0.00^{\mathrm{AB}}$ & $7.9 \pm 0.00^{\mathrm{A}}$ & $32500 \pm 0.00^{\mathrm{A}}$ & $7.64 \pm 0.00^{\mathrm{A}}$ \\
& $\mathrm{S} 2$ & $18.8 \pm 0.00^{\mathrm{A}}$ & $7.2 \pm 0.00^{\mathrm{B}}$ & $45000 \pm 0.00^{\mathrm{B}}$ & $6.50 \pm 0.00^{\mathrm{B}}$ \\
& $\mathrm{S} 3$ & $18.2 \pm 0.00^{\mathrm{B}}$ & $7.6 \pm 0.00^{\mathrm{AB}}$ & $40100 \pm 0.00^{\mathrm{AB}}$ & $6.85 \pm 0.00^{\mathrm{AB}}$ \\
\hline \multirow{3}{*}{ Winter } & $\mathrm{S} 1$ & $16.0 \pm 0.00^{\mathrm{AB}}$ & $8.7 \pm 0.00^{\mathrm{A}}$ & $32000 \pm 0.00^{\mathrm{A}}$ & $7.50 \pm 0.00^{\mathrm{A}}$ \\
& $\mathrm{S} 2$ & $17.2 \pm 0.00^{\mathrm{A}}$ & $7.5 \pm 0.00^{\mathrm{B}}$ & $43100 \pm 0.00^{\mathrm{B}}$ & $6.20 \pm 0.00^{\mathrm{B}}$ \\
& $\mathrm{S} 3$ & $15.5 \pm 0.00^{\mathrm{B}}$ & $8.4 \pm 0.00^{\mathrm{AB}}$ & $34000 \pm 0.00^{\mathrm{AB}}$ & $7.21 \pm 0.00^{\mathrm{AB}}$ \\
\hline \multirow{5}{*}{ Spring } & $\mathrm{S} 1$ & $17.9 \pm 0.00^{\mathrm{A}}$ & $8.6 \pm 0.00^{\mathrm{A}}$ & $39000 \pm 0.00^{\mathrm{A}}$ & $7.85 \pm 0.00^{\mathrm{A}}$ \\
& $\mathrm{S} 2$ & $18.8 \pm 0.00^{\mathrm{B}}$ & $8.1 \pm 0.00^{\mathrm{B}}$ & $44000 \pm 0.00^{\mathrm{B}}$ & $5.60 \pm 0.00^{\mathrm{B}}$ \\
& $\mathrm{S} 3$ & $18.2 \pm 0.00^{\mathrm{AB}}$ & $8.5 \pm 0.00^{\mathrm{AB}}$ & $41000 \pm 0.00^{\mathrm{AB}}$ & $7.10 \pm 0.00^{\mathrm{AB}}$ \\
\hline \multirow{5}{*}{ Summer } & $\mathrm{S} 1$ & $20.0 \pm 0.00^{\mathrm{A}}$ & $8.5 \pm 0.00^{\mathrm{A}}$ & $32300 \pm 0.00^{\mathrm{A}}$ & $8.00 \pm 0.00^{\mathrm{A}}$ \\
& $\mathrm{S} 2$ & $22.6 \pm 0.00^{\mathrm{AB}}$ & $7.9 \pm 0.00^{\mathrm{B}}$ & $47000 \pm 0.00^{\mathrm{B}}$ & $6.80 \pm 0.00^{\mathrm{B}}$ \\
& $\mathrm{S} 3$ & $23.46 \pm 0.00^{\mathrm{B}}$ & $8 \pm 0.00^{\mathrm{AB}}$ & $45500 \pm 0.00^{\mathrm{AB}}$ & $7.5 \pm 0.00^{\mathrm{AB}}$ \\
\hline
\end{tabular}

Different upper-case letters in the same column indicate differences between the studied stations for the four seasons $(\mathrm{p}<0.05)$

The spatial variation of TSS, $\mathrm{BOD}_{5}, \mathrm{COD}$, and $\mathrm{OM}$ in sea water are given in Table 3. The statistical analysis of these data revealed a significant difference between the control and the polluted stations, especially between S1 and S2 ( $<<0.05)$.

Table 3. Seasonal variation of total suspended solids, biological oxygen demand, chemical oxygen demand, and oxidizable matter $\left(\mathrm{mg} \mathrm{L}^{-1}\right)$ in the seawater at the three studied areas

\begin{tabular}{c|c|c|c|c|c}
\hline Season & Station & TSS & BOD & COD & OM \\
\hline \multirow{5}{*}{ Autumn } & S1 & $42.00 \pm 1.00^{\mathrm{A}}$ & $05.66 \pm 0.57^{\mathrm{A}}$ & $13.00 \pm 1.73^{\mathrm{A}}$ & $08.11 \pm 0.83^{\mathrm{A}}$ \\
& $\mathrm{S} 2$ & $55.66 \pm 1.15^{\mathrm{B}}$ & $45.00 \pm 5.00^{\mathrm{B}}$ & $83.00 \pm 4.35^{\mathrm{B}}$ & $57.67 \pm 4.58^{\mathrm{B}}$ \\
& $\mathrm{S} 3$ & $53.33 \pm 0.57^{\mathrm{AB}}$ & $32.66 \pm 2.51^{\mathrm{AB}}$ & $59.00 \pm 1.00^{\mathrm{AB}}$ & $41.44 \pm 2.01^{\mathrm{AB}}$ \\
\hline \multirow{5}{*}{ Winter } & $\mathrm{S} 1$ & $31.57 \pm 0.51^{\mathrm{A}}$ & $03.66 \pm 1.15^{\mathrm{A}}$ & $8.33 \pm 2.08^{\mathrm{A}}$ & $05.22 \pm 0.19^{\mathrm{A}}$ \\
& $\mathrm{S} 2$ & $127.33 \pm 0.57^{\mathrm{B}}$ & $31.33 \pm 1.53^{\mathrm{B}}$ & $75.67 \pm 3.51^{\mathrm{B}}$ & $46.11 \pm 1.95^{\mathrm{B}}$ \\
& $\mathrm{S} 3$ & $71.16 \pm 0.29^{\mathrm{AB}}$ & $25.33 \pm 2.52^{\mathrm{AB}}$ & $41.00 \pm 1.73^{\mathrm{AB}}$ & $30.56 \pm 1.71^{\mathrm{AB}}$ \\
\hline \multirow{5}{*}{ Spring } & $\mathrm{S} 1$ & $26.41 \pm 0.37^{\mathrm{A}}$ & $3.66 \pm 0.58^{\mathrm{A}}$ & $34.00 \pm 1.00^{\mathrm{A}}$ & $13.78 \pm 0.69^{\mathrm{A}}$ \\
& $\mathrm{S} 2$ & $86.26 \pm 0.12^{\mathrm{B}}$ & $37.00 \pm 1.73^{\mathrm{B}}$ & $70.67 \pm 1.53^{\mathrm{B}}$ & $48.22 \pm 0.84^{\mathrm{B}}$ \\
& $\mathrm{S} 3$ & $66.66 \pm 0.01^{\mathrm{AB}}$ & $30.33 \pm 0.75^{\mathrm{AB}}$ & $36.33 \pm 2.08^{\mathrm{AB}}$ & $32.33 \pm 1.00^{\mathrm{AB}}$ \\
\hline \multirow{5}{*}{ Summer } & $\mathrm{S} 1$ & $52.21 \pm 0.03^{\mathrm{A}}$ & $6.33 \pm 0.58^{\mathrm{A}}$ & $16.33 \pm 1.53^{\mathrm{A}}$ & $09.67 \pm 0.88^{\mathrm{A}}$ \\
& $\mathrm{S} 2$ & $40.00 \pm 0.01^{\mathrm{AB}}$ & $56.67 \pm 1.53^{\mathrm{B}}$ & $94.67 \pm 4.16^{\mathrm{B}}$ & $69.33 \pm 2.31^{\mathrm{B}}$ \\
& $\mathrm{S} 3$ & $33.77 \pm 0.22^{\mathrm{B}}$ & $41.33 \pm 1.53^{\mathrm{AB}}$ & $69.00 \pm 2.00^{\mathrm{AB}}$ & $50.56 \pm 1.68^{\mathrm{AB}}$ \\
\hline
\end{tabular}

Different upper-case letters in the same column indicate differences between the studied stations for the four seasons $(\mathrm{p}<0.05)$

The TSS values varied between 26.41 and $127.33 \mathrm{mg} \mathrm{L}^{-1}$. Particularly high levels were recorded at S2, especially during winter and spring, reaching 127.33 and $86.26 \mathrm{mg} \mathrm{L}^{-1}$, respectively. Medium concentrations were recorded at $\mathrm{S} 3$ that varied between 33.77 and $71.16 \mathrm{mg} \mathrm{L}^{-1}$, while the lowest concentrations were observed at $\mathrm{S} 1$ 
(Table 3). The highest BOD values were recorded at $\mathrm{S} 2\left(56.67 \mathrm{mg} \mathrm{L}^{-1}\right)$ and $\mathrm{S} 3$ (41.33 $\mathrm{mg} \mathrm{L}^{-1}$ ) both recorded during summer, while considerably lower values were recorded at $\mathrm{S} 1$ especially during winter $\left(3.66 \mathrm{mg} \mathrm{L}^{-1}\right)$. Likewise, maximum COD values were recorded during summer at $\mathrm{S} 2\left(94.67 \mathrm{mg} \mathrm{L}^{-1}\right)$, and the lowest values were found at $\mathrm{S} 1$ (Table 3). The same was true for $\mathrm{OM}$ which exhibited a minimum at $\mathrm{S} 1$ of $5.22 \mathrm{mg} \mathrm{L}^{-1}$ and a maximum at $\mathrm{S} 2\left(69.33 \mathrm{mg} \mathrm{L}^{-1}\right)$ recorder during winter and summer, respectively (Table 3).

Concentrations of nutrients ( $\mathrm{TN}, \mathrm{NH}_{4}-\mathrm{N}, \quad \mathrm{NO}_{3}-\mathrm{N}, \quad \mathrm{TP}$ and $\mathrm{PO}_{4}-\mathrm{P}$ ) exhibited significant differences, Also, between control station $\mathrm{S} 1$ and the other polluted station $\mathrm{S} 2(\mathrm{p}<0.05)($ Table 4).

Total nitrogen concentrations (TN) ranged from $6 \mathrm{mg} \mathrm{L}^{-1}$ at $\mathrm{S} 1$ to $12.97 \mathrm{mg} \mathrm{L}^{-1}$ at $\mathrm{S} 2$. The minimum value of ammonium was also recorded at $\mathrm{S} 1\left(0.12 \mathrm{mg} \mathrm{L}^{-1}\right)$ and the maximum at $\mathrm{S} 2\left(0.53 \mathrm{mg} \mathrm{L}^{-1}\right)$. However, elevated nitrate concentrations were found at S1 (Maximum: $4.41 \mathrm{mg} \mathrm{L}^{-1}$ ) in comparison to the other stations (Table 4).

For phosphorus compounds, the lowest concentrations of $\mathrm{PO}_{4}-\mathrm{P}$ were found at $\mathrm{S} 1$ in season autumn $\left(0.02 \mathrm{mg} \mathrm{L}^{-1}\right)$, whereas the highest concentration was measured at $\mathrm{S} 2$ in spring $\left(0.21 \mathrm{mg} \mathrm{L}^{-1}\right)$ (Table 4).

Table 4. Seasonal variation of $T N, N_{4}-N, N_{3}-N, T P$ and $P O_{4}-P\left(m g L^{-1}\right)$ in the seawater at the three studied areas

\begin{tabular}{c|c|c|c|c|c|c}
\hline Season & Station & $\mathbf{T N}$ & $\mathbf{N H}_{\mathbf{4}}-\mathbf{N}$ & $\mathbf{N O}_{3}-\mathbf{N}$ & $\mathbf{T P}$ & $\mathbf{P O}_{4}-\mathbf{P}$ \\
\hline \multirow{4}{*}{ Autumn } & $\mathrm{S} 1$ & $6.25 \pm 0.09^{\mathrm{A}}$ & $0.32 \pm 0.01^{\mathrm{A}}$ & $3.53 \pm 0.01^{\mathrm{A}}$ & $0.39 \pm 0.01^{\mathrm{A}}$ & $0.02 \pm 0.01^{\mathrm{A}}$ \\
& $\mathrm{S} 2$ & $9.27 \pm 0.04^{\mathrm{B}}$ & $0.53 \pm 0.01^{\mathrm{B}}$ & $2.20 \pm 0.01^{\mathrm{B}}$ & $0.52 \pm 0.01^{\mathrm{B}}$ & $0.06 \pm 0.01^{\mathrm{B}}$ \\
& $\mathrm{S} 3$ & $8.06 \pm 0.08^{\mathrm{AB}}$ & $0.50 \pm 0^{\mathrm{AB}}$ & $2.69 \pm 0.01^{\mathrm{AB}}$ & $0.42 \pm 0.01^{\mathrm{AB}}$ & $0.03 \pm 0.01^{\mathrm{AB}}$ \\
\hline \multirow{5}{*}{ Winter } & $\mathrm{S} 1$ & $6.00 \pm 0.04^{\mathrm{A}}$ & $0.06 \pm 0^{\mathrm{A}}$ & $4.41 \pm 0.02^{\mathrm{A}}$ & $0.38 \pm 0.01^{\mathrm{A}}$ & $0.06 \pm 0.01^{\mathrm{A}}$ \\
& $\mathrm{S} 2$ & $12.97 \pm 0.03^{\mathrm{B}}$ & $0.23 \pm 0^{\mathrm{B}}$ & $2.85 \pm 0.02^{\mathrm{B}}$ & $0.60 \pm 0.01^{\mathrm{B}}$ & $0.12 \pm 0.01^{\mathrm{B}}$ \\
& $\mathrm{S} 3$ & $7.91 \pm 0.05^{\mathrm{AB}}$ & $0.18 \pm 0^{\mathrm{AB}}$ & $3.52 \pm 0.01^{\mathrm{AB}}$ & $0.50 \pm 0.01^{\mathrm{AB}}$ & $0.07 \pm 0.01^{\mathrm{AB}}$ \\
\hline \multirow{5}{*}{ Spring } & $\mathrm{S} 1$ & $8.77 \pm 0.03^{\mathrm{A}}$ & $0.14 \pm 0^{\mathrm{A}}$ & $2.09 \pm 0.02^{\mathrm{A}}$ & $1.92 \pm 0.07^{\mathrm{A}}$ & $0.13 \pm 0.01^{\mathrm{A}}$ \\
& $\mathrm{S} 2$ & $10.87 \pm 0.13^{\mathrm{B}}$ & $0.23 \pm 0^{\mathrm{B}}$ & $1.33 \pm 0.02^{\mathrm{B}}$ & $7.20 \pm 0.05^{\mathrm{B}}$ & $0.21 \pm 0.01^{\mathrm{B}}$ \\
& $\mathrm{S} 3$ & $8.96 \pm 0.07^{\mathrm{AB}}$ & $0.17 \pm 0^{\mathrm{AB}}$ & $1.40 \pm 0.01^{\mathrm{AB}}$ & $2.90 \pm 0.05^{\mathrm{AB}}$ & $0.14 \pm 0.01^{\mathrm{AB}}$ \\
\hline \multirow{3}{*}{ Summer } & $\mathrm{S} 1$ & $9.64 \pm 0.10^{\mathrm{A}}$ & $0.12 \pm 0^{\mathrm{A}}$ & $1.79 \pm 0.01^{\mathrm{A}}$ & $5.20 \pm 0.03^{\mathrm{A}}$ & $0.04 \pm 0.01^{\mathrm{A}}$ \\
& $\mathrm{S} 2$ & $11.98 \pm 0.24^{\mathrm{B}}$ & $0.22 \pm 0^{\mathrm{B}}$ & $1.69 \pm 0.01^{\mathrm{B}}$ & $7.38 \pm 0.02^{\mathrm{B}}$ & $0.06 \pm 0.01^{\mathrm{B}}$ \\
& $\mathrm{S} 3$ & $10.3 \pm 0.14^{\mathrm{AB}}$ & $0.18 \pm 0.01^{\mathrm{AB}}$ & $1.70 \pm 0.01^{\mathrm{AB}}$ & $5.73 \pm 0.05^{\mathrm{AB}}$ & $0.05 \pm 0.01^{\mathrm{AB}}$ \\
\hline
\end{tabular}

Different upper-case letters in the same column indicate differences between the studied stations for the four seasons $(\mathrm{p}<0.05)$

\section{Heavy metals}

$\mathrm{Pb}, \mathrm{Cr}, \mathrm{Ni}$ and $\mathrm{Cd}$ contents of E. elongata were always below the detection limit at the different sites during the four seasons. In contrast, $\mathrm{Cu}$ and $\mathrm{Zn}$ were detected and their concentrations differed significantly between the studied stations throughout the study period ( $\mathrm{p}<0.05)$ (Table 5). The highest values were always recorded at $\mathrm{S} 2$, with up to $2.64 \mu \mathrm{g} \mathrm{g}^{-1} \mathrm{DW}$ and $0.99 \mu \mathrm{g} \mathrm{g}{ }^{-1} \mathrm{DW}$ of $\mathrm{Zn}$ and $\mathrm{Cu}$, respectively.

\section{Physiological parameters}

Table 6 shows the physiological parameters of the seaweed E. elongata. The statistical analysis detected significant differences for all the parameters between the 
control station S1 and the station S2 $(\mathrm{p}<0.05)$, except for TPC and axis length during winter.

Table 5. Seasonal variation of zinc and copper $\left(\mu g g^{-1}\right.$ dry weight) in E. elongata at the three studied areas

\begin{tabular}{c|c|c|c}
\hline Season & Station & Zn & Cu \\
\hline \multirow{3}{*}{ Autumn } & S1 & $1.13 \pm 0.003^{\mathrm{A}}$ & $0.04 \pm 0.002^{\mathrm{A}}$ \\
& S2 & $2.02 \pm 0.00^{\mathrm{B}}$ & $0.12 \pm 0.001^{\mathrm{B}}$ \\
& S3 & $1.41 \pm 0.001^{\mathrm{AB}}$ & $0.07 \pm 0.00^{\mathrm{AB}}$ \\
\hline \multirow{3}{*}{ Winter } & S1 & $1.31 \pm 0.002^{\mathrm{A}}$ & $0.07 \pm 0.002^{\mathrm{A}}$ \\
& S2 & $2.64 \pm 0.003^{\mathrm{B}}$ & $0.14 \pm 0.003^{\mathrm{B}}$ \\
& S3 & $1.50 \pm 0^{\mathrm{AB}}$ & $0.13 \pm 0.002^{\mathrm{AB}}$ \\
\hline \multirow{3}{*}{ Spring } & S1 & $1.94 \pm 0.002^{\mathrm{AB}}$ & $0.09 \pm 0.001^{\mathrm{A}}$ \\
& $\mathrm{S} 2$ & $2.03 \pm 0.001^{\mathrm{A}}$ & $0.17 \pm 0.001^{\mathrm{B}}$ \\
& $\mathrm{S} 3$ & $1.13 \pm 0.001^{\mathrm{B}}$ & $0.17 \pm 0.002^{\mathrm{AB}}$ \\
\hline \multirow{3}{*}{ Summer } & $\mathrm{S} 1$ & $0.96 \pm 0.001^{\mathrm{A}}$ & $0.09 \pm 0.00^{\mathrm{A}}$ \\
& $\mathrm{S} 2$ & $1.79 \pm 0.170^{\mathrm{B}}$ & $0.99 \pm 0.001^{\mathrm{B}}$ \\
& $\mathrm{S} 3$ & $1.31 \pm 0.001^{\mathrm{AB}}$ & $0.13 \pm 0.003^{\mathrm{AB}}$ \\
\hline
\end{tabular}

Different upper-case letters in the same column indicate differences between the studied stations for the four seasons $(\mathrm{p}<0.05)$

Samples from station S1 exhibited both the highest content of chlorophyll a (Maximum: $433.68 \mu \mathrm{g} \mathrm{g}^{-1} \mathrm{FW}$ during winter) and the longest thalli (maximum: $5.77 \mathrm{~cm}$ during summer), respectively. Lower values were found at S2, with $86.67 \mu \mathrm{g} \mathrm{g}^{-1} \mathrm{FW}$ during spring and $1.53 \mathrm{~cm}$ during summer, respectively. In contrast, the highest values of proline and GB were recorded at S2 $\left(54.72 \mathrm{mg} \mathrm{g}^{-1} \mathrm{DW}\right.$ and $869.39 \mu \mathrm{g} \mathrm{g}^{-1} \mathrm{DW}$, respectively), while their minimum was always recorded at $\mathrm{S} 1$ (Table 6). With the exception of spring TPC also always reached its minimum at S1 (absolute minimum: $477.17 \mu \mathrm{g} \mathrm{g}^{-1} \mathrm{DW}$ ). Seasonal maxima were always observed at S2 or S3 (absolute maxima: 2116.58 and $2794.83 \mu \mathrm{g} \mathrm{g}^{-1} \mathrm{DW}$, respectively).

Table 6. Seasonal variation of Chla ( $\mu \mathrm{g} g-1$ fresh weight), proline ( $m g$ g-1 dry weight), GB and TPC ( $\mu g \mathrm{~g}-1$ dry weight) and axis length $(\mathrm{cm})$ of E. elongata at the three studied areas

\begin{tabular}{|c|c|c|c|c|c|c|}
\hline Season & Station & Chla & GB & Proline & TPC & Axis length \\
\hline \multirow{3}{*}{ Autumn } & $\mathrm{S} 1$ & $315.85 \pm 0.44^{\mathrm{A}}$ & $291.16 \pm 10.27^{\mathrm{A}}$ & $21.35 \pm 0.47^{\mathrm{A}}$ & $477.17 \pm 15.62^{\mathrm{A}}$ & $5.60 \pm 0.26^{\mathrm{A}}$ \\
\hline & $\mathrm{S} 2$ & $182.83 \pm 0.25^{\mathrm{B}}$ & $704.76 \pm 06.23^{\mathrm{B}}$ & $54.72 \pm 0.99^{\mathrm{B}}$ & $657.81 \pm 21.29^{\mathrm{A}}$ & $3.60 \pm 0.10^{\mathrm{B}}$ \\
\hline & $\mathrm{S} 3$ & $216.53 \pm 0.42^{\mathrm{AB}}$ & $518.37 \pm 08.16^{\mathrm{AB}}$ & $32.10 \pm 0.72^{\mathrm{AB}}$ & $671.44 \pm 15.62^{\mathrm{A}}$ & $4.57 \pm 0.35^{\mathrm{AB}}$ \\
\hline \multirow{3}{*}{ Winter } & $\mathrm{S} 1$ & $433.68 \pm 0.80^{\mathrm{A}}$ & $303.40 \pm 13.12^{\mathrm{A}}$ & $06.67 \pm 0.13^{\mathrm{A}}$ & $1960.53 \pm 35.91^{\mathrm{A}}$ & $5.47 \pm 0.50^{\mathrm{A}}$ \\
\hline & $\mathrm{S} 2$ & $180.94 \pm 0.19^{\mathrm{B}}$ & $855.78 \pm 08.50^{\mathrm{B}}$ & $17.07 \pm 0.26^{\mathrm{B}}$ & $2116.58 \pm 46.86^{\mathrm{AB}}$ & $4.43 \pm 1.04^{\mathrm{A}}$ \\
\hline & $\mathrm{S} 3$ & $232.92 \pm 0.41^{\mathrm{AB}}$ & $629.93 \pm 06.23^{\mathrm{AB}}$ & $10.09 \pm 0.27^{\mathrm{AB}}$ & $2794.83 \pm 56.31^{\mathrm{B}}$ & $4.03 \pm 0.15^{\mathrm{A}}$ \\
\hline \multirow{3}{*}{ Spring } & $\mathrm{S} 1$ & $254.04 \pm 0.36^{\mathrm{A}}$ & $521.09 \pm 06.23^{\mathrm{A}}$ & $14.08 \pm 1.61^{\mathrm{A}}$ & $961.15 \pm 27.05^{\mathrm{AB}}$ & $5.00 \pm 0.50^{\mathrm{A}}$ \\
\hline & $\mathrm{S} 2$ & $86.67 \pm 0.56^{\mathrm{B}}$ & $869.39 \pm 08.16^{\mathrm{B}}$ & $39.77 \pm 1.61^{\mathrm{B}}$ & $1428.09 \pm 50.44^{\mathrm{A}}$ & $2.57 \pm 0.12^{\mathrm{B}}$ \\
\hline & $\mathrm{S} 3$ & $189.20 \pm 0.31^{\mathrm{AB}}$ & $606.80 \pm 08.50^{\mathrm{AB}}$ & $23.94 \pm 0.61^{\mathrm{AB}}$ & $576.01 \pm 56.31^{\mathrm{B}}$ & $3.50 \pm 0.50^{\mathrm{AB}}$ \\
\hline \multirow{3}{*}{ Summer } & $\mathrm{S} 1$ & $328.75 \pm 1.26^{\mathrm{A}}$ & $533.33 \pm 10.27^{\mathrm{A}}$ & $12.79 \pm 1.38^{\mathrm{A}}$ & $562.38 \pm 30.68^{\mathrm{A}}$ & $5.77 \pm 0.38^{\mathrm{A}}$ \\
\hline & $\mathrm{S} 2$ & $121.37 \pm 0.40^{\mathrm{B}}$ & $858.50 \pm 04.71^{\mathrm{B}}$ & $39.18 \pm 1.38^{\mathrm{B}}$ & $749.83 \pm 51.46^{\mathrm{AB}}$ & $1.53 \pm 0.38^{\mathrm{B}}$ \\
\hline & S3 & $157.74 \pm 0.56^{\mathrm{AB}}$ & $801.36 \pm 04.71^{\mathrm{AB}}$ & $21.99 \pm 0.69^{\mathrm{AB}}$ & $940.70 \pm 30.68^{\text {B }}$ & $2.60 \pm 0.26^{\mathrm{AB}}$ \\
\hline
\end{tabular}




\section{Statistical analysis}

As shown on the Pearson correlation matrix (Table 7), parameters that reflect organic pollution (BOD, COD and OM) exhibit strong positive correlations with TN, GB and proline $(r>0.7)$, but negative correlations with Chla and morphometry $(r>-0.7)$. However, the organic parameters show mainly weak relationships with the remaining parameters (e.g., TPC, $\mathrm{PO}_{4}$ ).

Table 7. Pearson correlation matrix between the parameters studied

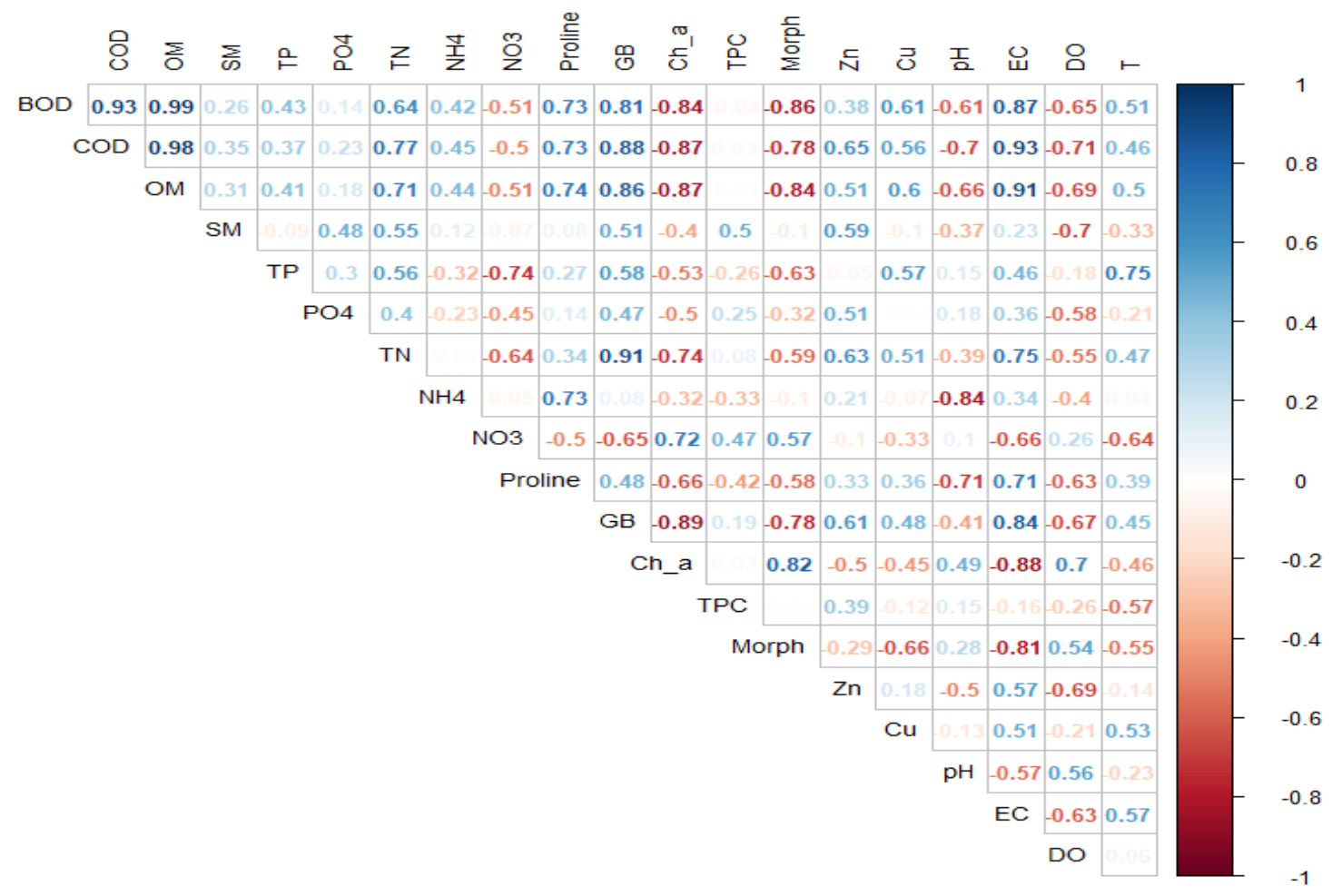

Principal Component Analysis (PCA) of the physiological parameters (Morphometry, Chlorophyll a, Proline, and Glycine betaine (GB) combined with the physico-chemical parameters (T, pH, EC, TSS, DO, BOD, COD, and OM) and heavy metal concentrations $(\mathrm{Cu}$ and $\mathrm{Zn})$ allowed the identification of two principal components that together explain more than $75.72 \%$ of the overall variability of samples (Table 8). Our PCA analysis allows to identify the most representative variables that distinguish the studied stations (Fig. 3). It demonstrates that BOD, COD, OM, Proline, GB, and EC are correlated positively with the PC1 $(0.941 ; 0.975 ; 0.974 ; 0.775 ; 0.892 ; 0.934$, respectively). In contrast, the algal axis length, Chla and DO are negatively correlated with the PC1 (0.834; $0.891 ; 0.779$, respectively). TSS and $\mathrm{Cu}$ together correlate with $\mathrm{PC} 2$, while $\mathrm{T}$ and $\mathrm{Zn}$ together correlate along PC1. However, both heavy metals did not correlate particularly well with the algal physiological parameters (Table 8).

\section{Discussion}

Our study aimed for the first time to use physiological parameters of Ellisolandia elongata in combination with macroalgal biodiversity and physico-chemical 
parameters for the assessment of coastal pollution. Three stations were investigated for this purpose. $\mathrm{S} 1$ is the reference site, while $\mathrm{S} 2$ and $\mathrm{S} 3$ received domestic and industrial wastewaters, containing organic and inorganic pollutants. The impact of pollution was partly reflected by the algal biodiversity existing in the studied area. Likewise, we found at the reference station S1 which suffers only small anthropogenic impacts the highest biodiversity of seaweeds. Genera such as Cystoseira, Bifurcaria and Saccorhiza were present at this site, which typically indicate small anthropogenic impacts or non-affected areas (Munda, 1980; Riadi, 1998; Diĺez et al., 1999; Arévalo et al., 2007; Ballesteros et al., 2007; Orfanidis et al., 2011; Biskup et al., 2014; Rubal et al., 2014; Celis-Plá et al., 2016; Boundir et al., 2019). In contrast, a dramatic decrease of diversity was observed in sites S2 and S3, located in the urban area close to places where domestic and some industrial waste waters are discharged. Several authors have proven the disappearance of mostly perennial seaweeds including the associated or epiphytic species due to pollution impact (Munda, 1982; Schramm, 1999; Sava et al., 2011). Our finding is in agreement with these earlier reports from other areas and it also generally supports the work of Chouikh et al. (2019) that was based on the assessment of polychaete distribution and conducted in the same study area. Indeed, many epiphytic algae inventoried at S1 (e.g. Aglaothamnion tripinnatum, Polysiphonia atlantica, P. elongata, Lomentaria articulata, Plocamium raphelisianum, $P$. cartilagineum) were not found at S2 and S3. In contrast, it has been noticed that species known to indicate pollution, like Caulacanthus ustulatus, Codium tomentosum, and other species belonging to the Cladophora and Ulva genera, were dominant at the last two stations. These findings are in accordance with literature (Jupp, 1976; DÍez et al., 1999; Godeh et al., 2010; Orfanidis et al., 2011). In order to support our conclusions an established indicator of pollution was calculated, which is the mean ratio of red algal and green algal species diversity in a given area (the socalled corrected R/C index). It is based on the fact that the number of Rhodophyceae is generally higher in less polluted areas, whereas Chlorophyceae are more diverse in polluted habitats, pollution is coupled to a decrease of the ratio (Sfriso et al., 2006; Orfanidis et al., 2011). At $\mathrm{S} 1$, the $\mathrm{R} / \mathrm{C}$ ratio was always above 2 (on average 3.84), indicating a high percentage of Rhodophyceae, while at the stations S2 and S3 it never exceeded 0.91 and 1.37, respectively. Moreover, a stronger dominance of opportunistic species was observed at S2 (78\%) and S3 (66\%), as compared to S1 $(42 \%)$. Again, similar observations at differently impacted sites have been reported from other coastal areas (Sfriso et al., 2009).

Nitrate, ammonium and orthophosphate are essential macronutrients for algal growth and stress management (Celis-Plá et al., 2014). However, excess concentrations can have a negative impact, as they can generate additional stress and thus inhibit growth (Guerra-García and Koonjul, 2005; Nassar et al., 2015). The macronutrients of coastal areas are often a main factor that drives changes in the structural composition of algal communities (McGlathery et al., 2007; Leterme et al., 2014; Nassar et al., 2015). Such nutrients are present in natural environments, but their concentration near urban areas is usually elevated (Nixon, 1995; Scavia and Bricker, 2006). In the present study, the physiology of E. elongata did not correlate significantly with most of the inorganic parameters. This indicates that the state of this seaweed is not really affected by the nutrient concentration at the study site varied between 6 and 12 and 0.3 and 7 for TN and TP $\mathrm{mg} \mathrm{L}^{-1}$, respectively, which has also been observed by some other authors (Schaffelke, 1999; Boundir et al., 2019). 


$$
-7729-
$$

Table 8. Loading vectors of physiological parameters and heavy metal concentrations in E. elongata and physicochemical parameters in the study areas along principal component axes 1 and 2

\begin{tabular}{c|c|c}
\hline \multirow{2}{*}{ Parameter } & \multicolumn{2}{|c}{ Component } \\
\cline { 2 - 3 } & $\mathbf{1}$ & $\mathbf{2}$ \\
\hline BOD & 0.941 & 0.136 \\
COD & 0.975 & -0.011 \\
OM & 0.974 & 0.071 \\
TSS & 0.383 & -0.724 \\
Proline & 0.775 & 0.135 \\
GB & 0.892 & -0.091 \\
Chla & -0.891 & 0.031 \\
Morphometry & -0.834 & -0.295 \\
Zn & 0.526 & 0.554 \\
Cu & 0.607 & -0.540 \\
pH & -0.658 & 0.229 \\
EC & 0.934 & 0.091 \\
DO & -0.779 & 0.472 \\
T & 0.473 & 0.676 \\
Variance $(\%)$ & 41.85 & 33.86 \\
\hline Cumulative $(\%)$ & 41.85 & 75.72 \\
\hline
\end{tabular}

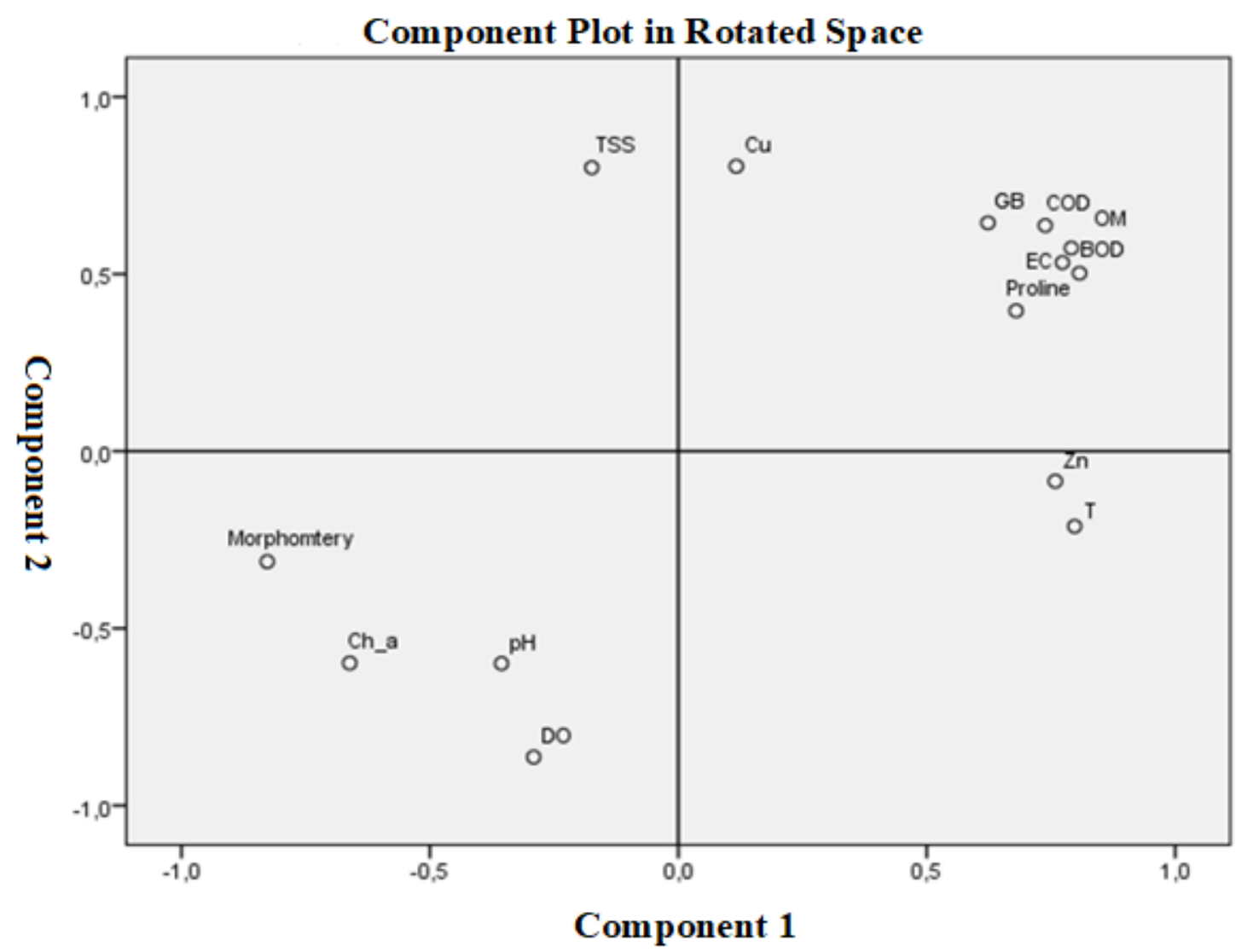

Figure 3. Component plot in rotated space for the parameters studied. TSS: total suspended solids; T: temperature; EC: electrical conductivity; DO: dissolved oxygen; BOD: biological oxygen demand; COD: chemical oxygen demand; OM: oxidizable matter; Ch a: chlorophyll a;

GB: glycine betaine; $\mathrm{Zn:}$ zinc; $\mathrm{Cu}$ : copper 
A significant increase of tissue concentrations of GB and proline in E. elongata and a decrease of Chla content, DO and algal axis length was observed together with an increase in concentrations of EC, BOD, COD and OM at the polluted stations S2 and S3. These results of our PCA analysis are in accordance with our expectation of an impact of organic pollution on E. elongata's physiology. Overall, algal length, chla and $\mathrm{DO}$ values were higher in $\mathrm{S} 1$ than in $\mathrm{S} 2$ and $\mathrm{S} 3$, which shows that E. elongata was affected by pollution in the two last stations.

Previous studies have demonstrated that pollution stress causes a decrease of chlorophyll contents, reducing algal photosynthetic activities, and correspondingly a reduction of production of dissolved oxygen, which can be decreased if pollution comes along with increased input of organic matter and subsequent degradation processes (Sawyer and McCarty, 1978). Additionally, some authors have shown that the pollution affects also the algal size (Doblin and Clayton, 1995; Coelho et al., 2000; Morin, 2006). However, toxic heavy metals $(\mathrm{Cd}, \mathrm{Pb}$ and $\mathrm{Cr})$ were not detected in E. elongata. Moreover, the $\mathrm{Zn}$ and $\mathrm{Cu}$ concentrations observed are much lower than those reported by other authors in calcareous red seaweeds (Malea et al., 1994; Abdallah et al., 2006; Dadolahi-sohrab et al., 2011; Khaled et al., 2014) and cannot be expected to be toxic. Working in our study area, some authors already demonstrated that brown algae such as Carpodesmia tamariscifolia (as Cystoseira tamariscifolia) and Saccorhiza polyschides are significantly more contaminated with heavy metals at sites S2 and S3 than at site S1 (Cherifi et al., 2018; Boundir et al., 2019). However, certain studies have already shown that calcareous Rhodophyceae have a reduced capacity for biosorption of metals as compared to other algal groups (Jordanova et al., 1999; Benguedda-Rahal, 2012). Bioaccumulation of heavy metals may be affected by many factors, such as $\mathrm{pH}$, the binding capacity of various sediment components and the specific affinity of species for heavy metals. This binding capacity is largely dependent on the nature and chemical composition of cell wall components. The latter might be a main reason for the findings at the study area, given that brown algae generally exhibit a greater ability to accumulate metals than red and green algae (Leonardi and Vasquez, 1999; Davis et al., 2000; Kaimoussi et al., 2002; Abdallah et al., 2006; Benkdad et al., 2011; Mouradi et al., 2014).

While the inorganic pollution parameters had no impact on the physiological parameters of E. elongata at the study sites there was a clear correlation with the parameters indicating organic pollution like $\mathrm{BOD}_{5}, \mathrm{COD}$, OM. The highest concentrations of $\mathrm{BOD}_{5}, \mathrm{COD}$, and $\mathrm{OM}$ were always recorded in the polluted areas $\mathrm{S} 2$ and S3 which were elevated by a factor 5-10 compared to the unpolluted site S1. That organic matter is an indicator for pollution by discharge of wastewater was shown before (Sawyer and McCarty, 1978; Broecker and Peng, 1983; El-Sonbati et al., 2012; Er-Raioui et al., 2012; El-zeiny et al., 2016). This does not explain, why and how the organic matter affected the seaweed. There are two possible explanations: the organic matter load included substances which were toxic or the organic matter enhanced heterotrophic degradation processes which affected the primary production. The latter can be ruled out because although $>50 \%$ of the COD was biologically degradable the parameters like temperature, $\mathrm{pH}, \mathrm{DO}$ and conductivity did not differ significantly at all study sites and during all season. It is thus highly possible that toxic organic pollutants entered the coastal areas with the discharge of waste water. Because this kind of pollution would have otherwise remained undetected, our approach to use physiological responses of E. elongata as indicators of variable kinds of pollution was verified. This is 
in accordance with a growing interest in using seaweeds for the biomonitoring of different kinds of pollution (Pereira et al., 2009; García-Seoane et al., 2018, 2019; Roleda et al., 2019). A number of researchers identified that stressful conditions of seaweeds induce the decrease of chlorophyll contents, algal size and also proline and GB accumulations (Table 9). Emphasis should be put on the development of methods which allow to indentify the type of pollution too.

Table 9. Main factors causing algal stress

\begin{tabular}{c|c|c}
\hline Stress factors & Algal species & References \\
\hline Solar radiation & Ellisolandia elongata & Häder et al. (1997) \\
Thermal stress & Ellisolandia elongata & Nannini et al. (2015) \\
Acidification effect & Lithophyllum incrustans & Noisette et al. (2013) \\
Light and desiccation stress & Ulva linza & Guan et al. (2016) \\
Heavy metals & Cystoseira tamariscifolia & Boundir et al. (2019) \\
Organic pollution & Ellisolandia elongata & Current study \\
\hline
\end{tabular}

\section{Conclusion}

The present work evaluated the pollution degree in the Essaouira city coastal area (Morocco) by using, for the first time, the calcareous seaweed Ellisolandia elongata (Rhodophyta) as a bioindicator. Our results show that organic pollution is the main factor that affects the macroalgae diversity at the Essaouira coast. A dramatic decrease of seaweed biodiversity has been noticed in the Bab Doukkala (S2) and Port (S3) stations where urban wastewater is discharged without any treatment, causing pollution. Several species reflecting these polluted conditions have been identified in these two stations and not in the reference station of My Bouzerktoune (S1). The pollution impact is more evident at S2 than at S3. Physico-chemical parameters and inorganic pollution did not show a real impact on the algal biodiversity and the physiological state of $E$. elongata. Thus, this red alga could be used as a tool to monitor organic pollution in Morocco and might be applicable as a bioindicator also in other areas. Further studies should be undertaken on other macroalgae and on other stressful conditions in order to support these results.

\section{REFERENCES}

[1] Abdallah, A. M. A., Abdallah, M. A., Beltagy, A., Siam, E. (2006): Contents of heavy metals in marine algae from Egyptian Red Sea coast. - Toxicological and Environmental Chemistry 88(1): 9-22.

[2] Abdel, A., Abdel, H. (2015): Changes in growth and some biochemical parameters of maize plants irrigated with sewage water. - Austin Journal of Plant Biology 1(1): 1-5.

[3] Adey, W. H., Macintyre, I. G. (1973): Crustose coralline algae: a re-evaluation in the geological sciences. - Bulletin of the Geological Society of America 84(3): 883-904.

[4] AFNOR NF EN ISO 6878. Avril (2005): Qualité de l'eau. Dosage du phosphore: méthode spectrophotométrique au molybdate d'ammonium (indice de classement T90023).

[5] AFNOR NF T90-012. Août (1975): Dosage des nitrates.

[6] AFNOR NF T90-103. Décembre (1975): Détermination de la demande biochimique en oxygène DBO. 
[7] AFNOR NF EN ISO 11905-1. Juillet (1998): Qualité de l'eau. Dosage de l'azote. Partie 1: Méthode par minéralisation oxydante au peroxodisulfate (indice de classement T 90061).

[8] AFNOR NF T90-015-2. Janvier (2000): Qualité de l'eau. Dosage de l'ammonium. Partie 2: méthode spectrométrique au bleu d'indophénol.

[9] AFNOR NF T90-101. Février (2001): Détermination de la demande chimique en oxygène DCO.

[10] AFNOR NF T90-022. Avril (2005): Dosage des orthophosphates.

[11] Agbaire, P. O. (2016): Impact of air pollution on proline and soluble sugar content pf selected plant species. - Chemistry and Materials Research 8(5): 72-76.

[12] Ali, G., Srivastava, P. S., Iqbal, M. (1999): Proline accumulation, protein pattern and photosynthesis in Bacopa monniera regenerants grown under $\mathrm{NaCl}$ stress. - Biologia Plantarum 42(1): 89-95.

[13] Alia, Saradhi, P. P. (1991): Proline Accumulation Under Heavy Metal Stress. - Journal of Plant Physiology 138(5): 554-558.

[14] Amin, B., Ismail, A., Arshad, A., Yap, C. K., Kamarudin, M. S. (2009): Anthropogenic impacts on heavy metal concentrations in the coastal sediments of Dumai, Indonesia. Environmental Monitoring and Assessment 148(1-4): 291-305.

[15] Amirjani, M. R. (2010): Effect of salinity stress on growth, mineral composition, proline content, antioxidant enzymes of soybean. - American Journal of Plant Physiology 5(6): 350-360.

[16] Amna, Ali, N., Masood, S., Mukhtar, T., Kamran, M. A., Rafique, M., Munis, M. F. H., Chaudhary, H. J. (2015): Differential effects of cadmium and chromium on growth, photosynthetic activity, and metal uptake of Linum usitatissimum in association with Glomus intraradices. - Environmental Monitoring and Assessment 187(6): 311.

[17] Anbazhagan, M., Krishnamurthy, R., Bhagwat, K. A. (1988): Proline: an enigmatic indicator of air pollution tolerance in rice cultivars. - Journal of Plant Physiology 133(1): 122-123.

[18] Ar Gall, E., Connan, S. (2004): Echantillonnage des macroalgues Intertidal - substrats durs. - REBENT REseau BENThique: 1-11.

[19] Arévalo, R., Pinedo, S., Ballesteros, E. (2007): Changes in the composition and structure of Mediterranean rocky-shore communities following a gradient of nutrient enrichment: descriptive study and test of proposed methods to assess water quality regarding macroalgae. - Marine Pollution Bulletin 55(1-6): 104-113.

[20] Ballesteros, E., Torras, X., Pinedo, S., García, M., Mangialajo, L., de Torres, M. (2007): A new methodology based on littoral community cartography dominated by macroalgae for the implementation of the European Water Framework Directive. - Marine Pollution Bulletin 55(1-6): 172-180.

[21] Bazairi, H., Harmelin, J. G., Turpin, Y., Aghori, A. (2010): Caractérisation des peuplements marins de l'archipel de Mogador (Atlantique, MAROC). - Rapport du programme de coopération entre l'Initiative des Petites Îles de Méditerranée du Conservatoire de l'espace et littoral des rivages lacustres (République Française) et le Haut-Commissariat aux Eaux et Forêts et à la Lutte Contre la Désertification.

[22] Benguedda, R. (2012): Contribution à l'étude de la bioaccumulation métallique dans les sédiments et différents maillons de la chaine trophique du littoral extrême ouest algérien. - PhD Thesis Doctoral, Telmcen University, Algeria.

[23] Benkdad, A., Laissaoui, A., Tornero, M. V., Benmansour, M., Chakir, E., Garrido, I. M., Moreno, J. B. (2011): Trace metals and radionuclides in macroalgae from Moroccan coastal waters. - Environmental Monitoring and Assessment 182(1-4): 317-324.

[24] Bibi, S., Khan, S., Taimur, N., Daud, M. K., Azizullah, A. (2019): Responses of morphological, physiological, and biochemical characteristics of maize (Zea mays L.) seedlings to atrazine stress. - Environmental Monitoring and Assessment 191(12): 717. 
[25] Biskup, S., Bertocci, I., Arenas, F., Tuya, F. (2014): Functional responses of juvenile kelps, Laminaria ochroleuca and Saccorhiza polyschides, to increasing temperatures. Aquatic Botany 113: 117-122.

[26] Boundir, Y., Hasni, M., Rafik, F., Sabri, H., Bahammou, N., Cheggour, M., Achtak, H., Cherifi, O. (2019): First study of the ecological status in the Atlantic coast of Morocco using the brown seaweed Cystoseira tamariscifolia. - Applied Ecology and Environmental Research 17(6): 14315-14331.

[27] Broecker, W. S., Peng, T. H. (1983): Tracers in the sea. - Radiocarbon 24(3): b1-b2.

[28] Carillo, P., Mastrolonardo, G., Nacca, F., Parisi, D., Verlotta, A., Fuggi, A. (2008): Nitrogen metabolism in durum wheat under salinity: accumulation of proline and glycine betaine. - Functional Plant Biology 35(5): 412.

[29] Celis-Plá, P. S. M., Martínez, B., Quintano, E., García-Sánchez, M., Pedersen, A., Navarro, N. P., Copertino, M. S., Mangaiyarkarasi, N., Mariath, R., Figueroa, F. L., Korbee, N. (2014): Short-term ecophysiological and biochemical responses of Cystoseira tamariscifolia and Ellisolandia elongata to environmental changes. - Aquatic Biology 22: 227-243.

[30] Celis-Plá, Paula, S. M., Bouzon, Z. L., Hall-Spencer, J. M., Schmidt, E. C., Korbee, N., Figueroa, F. L. (2016): Seasonal biochemical and photophysiological responses in the intertidal macroalga Cystoseira tamariscifolia (Ochrophyta). - Marine Environmental Research 115: 89-97.

[31] Cherifi, O., Sabri, H., Gharmali, A., Maarouf, A., Hasni, M., Cherifi, K., Ait-Hman, A., Derhem, A., Bahammou, N., Sbihi, K. (2018): Variation spatio-temporelle des métaux traces $(\mathrm{Cr}, \mathrm{Cu}, \mathrm{Pb}, \mathrm{Zn})$ chez la laminariale Saccorhiza polyschides au niveau du littoral de la région d'Essaouira. - SMETox Journal 1(1): 53-58.

[32] Chouikh, N., Gillet, P., Langston, W. J., Cheggour, M., Maarouf, A., Mouabad, A. (2019): First investigation of the composition and spatial distribution of polychaete feeding guilds from essaouira protected coastal area (Atlantic Coast of Morocco). Applied Ecology and Environmental Research 17(2): 3231-3249.

[33] Coelho, S. M., Rijstenbil, J. W., Brown, M. T. (2000): Impacts of anthropogenic stresses on the early development stages of seaweeds. - Journal of Aquatic Ecosystem Stress and Recovery 7(4): 317-333.

[34] Dadolahi-sohrab, A., Nikvarz, A., Mohamad, S., Nabavi, B., Safahyeh, A., Ketalmohseni, M. (2011): Environmental Monitoring of Heavy Metals in Seaweed and Associated Sediment from the Strait of Hormuz, I. R. Iran. - World Journal of Fish and Marine Sciences 3(6): 576-589.

[35] Davis, T. A., Volesky, B., Vieira, R. H. S. F. (2000): Sargassum seaweed as biosorbent for heavy metals. - Water Research 34(17): 4270-4278.

[36] De Faveri, C., Scherner, F., Farias, J., De Oliveira, E. C., Horta, P. A. (2010): Temporal changes in the seaweed flora in Southern Brazil and its potential causes. - Pan-American Journal of Aquatic Sciences 5(2): 350-357.

[37] Di Martino, C., Delfine, S., Pizzuto, R., Loreto, F., Fuggi, A. (2003): Free amino acids and glycine betaine in leaf osmoregulation of spinach responding to increasing salt stress. - New Phytologist 158(3): 455-463.

[38] DÍez, I., Secilla, A., Santolaria, A., Gorostiaga, J. M. (1999): Phytobenthic intertidal community structure along an environmental pollution gradient. - Marine Pollution Bulletin 38(6): 463-472.

[39] Doblin, M. A., Clayton, M. N. (1995): Effects of secondarily-treated sewage effluent on the early life-history stages of two species of brown macroalgae: Hormosira banksii and Durvillaea potatorum. - Marine Biology 122(4): 689-698.

[40] El-adl, M. F., El-katony, T. M., Bream, A. S. (2017): Effect of sewage pollution on macroalgal diversity and heavy metal accumulation along Al-Hanyaa Coastline, Libya. Advances in Environmental Biology 11(2): 52-59. 
[41] El-Sonbati, A. M., El-zeiny, A. M., El-gammal, M. I., Ibrahim, M. S. (2012): Assessment of some water pollutants at New Damietta. - Journal of Environmental Science 41(2): 267-282.

[42] El-zeiny, A., Gad, A.-A., El-Gammal, M. (2016): Geospatial techniques for detection of vulnerable areas to contamination at Damietta. - Journal of Environmental Science 5.

[43] Er-Raioui, H., Khannous, S., Ould Mohamd Cheihk, M., Mhamada, M., Bouzid, S. (2012): The Moroccan Mediterranean coastline: a potential threatened by the urban discharges. - The Open Environmental Pollution \& Toxicology Journal 3(1): 23-36.

[44] Erdoğrul, Ö., Erbilir, F. (2007): Heavy metal and trace elements in various fish samples from Sir Dam Lake, Kahramanmaraş, Turkey. - Environmental Monitoring and Assessment 130(1-3): 373-379.

[45] Fariduddin, Q., Khalil, R. R. A. E., Mir, B. A., Yusuf, M., Ahmad, A. (2013): 24Epibrassinolide regulates photosynthesis, antioxidant enzyme activities and proline content of Cucumis sativus under salt and/or copper stress. - Environmental Monitoring and Assessment 185(9): 7845-7856.

[46] Fatma, T., Khan, M. A., Choudhary, M. (2007): Impact of environmental pollution on cyanobacterial proline content. - Journal of Applied Phycology 19: 625-629.

[47] García-Seoane, R., Fernández, J. A., Varela, Z., Real, C., Boquete, M. T., Aboal, J. R. (2019): Sampling optimization for biomonitoring metal contamination with marine macroalgae. - Environmental Pollution 255(3): 113349.

[48] García-Seoane, R., Fernández, J. A., Villares, R., Aboal, J. R. (2018): Use of macroalgae to biomonitor pollutants in coastal waters: optimization of the methodology. - Ecological Indicators 84: 710-726.

[49] Godeh, M. M., Said, A. A., Zarmouh, M. M., El-Menifi, F. O. (2010): Marine Chlorophyta of Benghazi Coasts, Libya. - Journal of Science and Its Applications 4(1): 7-13.

[50] Grieve, C. M., Grattan, S. R. (1983): Rapid assay for determination of water soluble quaternary ammonium. - Plant and Soil 70: 303-307.

[51] Gu, D., Zhang, Y., Fu, J., Zhang, X. (2007): The landscape pattern characteristics of coastal wetlands in Jiaozhou Bay under the impact of human activities. - Environmental Monitoring and Assessment 124(1-3): 361-370.

[52] Guan, Z., Mou, S., Zhang, X., Xu, D., Fan, X., Wang, Y., Wang, D., Ye, N. (2016): Identification and expression analysis of four light harvesting-like (Lhc) genes associated with light and desiccation stress in Ulva linza. - Journal of Experimental Marine Biology and Ecology 478: 10-15.

[53] Guerra-García, J. M., Koonjul, M. S. (2005): Metaprotella sandalensis (Crustacea: Amphipoda: Caprellidae): a bioindicator of nutrient enrichment on coral reefs? Environmental Monitoring and Assessment 104(1-3): 353-367.

[54] Guiry, M. D., Guiry, G. M. (2020): AlgaeBase. - World-Wide Electronic Publication, National University of Ireland, Galway.

[55] Häder, D. P., Lebert, M., Flores-Moya, A., Jiménez, C., Mercado, J., Salles, S., Aguilera, J., Figueroa, F. L. (1997): Effects of solar radiation on the photosynthetic activity of the red alga Corallina elongata Ellis et Soland. - Journal of Photochemistry and Photobiology B: Biology 37(3): 196-202.

[56] Harley, C., Randall Hughes, A., Hultgren, K., Miner, B., Sorte, C., Thornber, C., Rodriguez, L. F., Tomanek, L., Williams, S. (2006): The impacts of climate change in coastal marine systems. - Ecology Letters 9(2): 228-241.

[57] Hong, J. H., Semprucci, F., Jeong, R., Kim, K., Lee, S., Jeon, D., Yoo, H., Kim, J., Kim, J., Yeom, J., Lee, S., Lee, K., Lee, W. (2020): Meiobenthic nematodes in the assessment of the relative impact of human activities on coastal marine ecosystem. - Environmental Monitoring and Assessment 192(2): 81. 
[58] Huertas Emma, I., Rouco, M., López-Rodas, V., Costas, E. (2011): Warming will affect phytoplankton differently: evidence through a mechanistic approach. - Proceedings of the Royal Society B: Biological Sciences 278(1724): 3534-3543.

[59] Islam, M. S., Tanaka, M. (2004): Impacts of pollution on coastal and marine ecosystems including coastal and marine fisheries and approach for management: a review and synthesis. - Marine Pollution Bulletin 48(7-8): 624-649.

[60] Jeffrey, S. W., Humphrey, G. F. (1975): New spectrophotometric equations for determining chlorophylls a, b, c1 and c2 in higher plants, algae and natural phytoplankton. - Biochemie Und Physiologie Der Pflanzen 167(2): 191-194.

[61] Jordanova, A., Strezov, A., Ayranov, M., Petkov, N., Stoilova, T. (1999): Heavy metal assessment in algae, sediments and water from the Bulgarian Black Sea coast. - Water Science and Technology 39(8): 207-212.

[62] Jupp, B. P. (1976): The effects of organic pollution on benthic organisms near Marseille. - International Journal of Environmental Studies 10(1): 119-123.

[63] Kaimoussi, A., Chafik, A., Mouzdahir, A., Bakkas, S. (2002): Diagnosis on the state of healthiness, quality of the coast and biological resources "case of the Moroccan Atlantic coast" (City of El Jadida). - Comptes Rendus Biologies 325(3): 253-260.

[64] Khairallah, Y., Houri, T., Osta, B., Romanos, D., Haddad, G. (2018): Biochemical profile of non-enzymatic stress markers in the plant species "Urginea maritima" in a Mediterranean natural reserve exposed to oxidative stress. - Environmental Monitoring and Assessment 190(6): 354.

[65] Khaled, A., Hessein, A., Abdel-Halim, A. M., Morsy, F. M. (2014): Distribution of heavy metals in seaweeds collected along Marsa-Matrouh beaches, Egyptian Mediterranean Sea. - Egyptian Journal of Aquatic Research 40(4): 363-371.

[66] Koivikko, R., Loponen, J., Honkanen, T., Jormalainen, V. (2005): Contents of soluble, cell-wall-bound and exuded phlorotannins in the brown alga Fucus vesiculosus, with implications on their ecological functions. - Journal of Chemical Ecology 31(1): 195212.

[67] Lamalakshmi, D. E., Kumar, S., Basanta, S. T., Sharma, S. K., Beemrote, A., Premabati, D. C., Chongtham, S. K., Chongtham, H. S., Yumlembam, R. A., Haribhushan, A., Prakash, N., Shabir, H. W. (2017): Adaptation Strategies and Defence Mechanisms of Plants During Environmental Stress: - In: Ghorbanpour, A. V. (ed.) Medicinal Plants and Environmental Challenges. Springer International, New York.

[68] Leonardi, P. I., Vasquez, J. A. (1999): Effects of copper pollution on the ultrastructure of Lessonia spp. - Hydrobiologia 398/399(0): 375-383.

[69] Leterme, S. C., Jendyk, J. G., Ellis, A. V., Brown, M. H., Kildea, T. (2014): Annual phytoplankton dynamics in the Gulf Saint Vincent, South Australia, in 2011. Oceanologia 56(4): 757-778.

[70] Liang, X., Zhang, L., Natarajan, S. K., Becker, D. F. (2013): Proline mechanisms of stress survival. - Antioxidants and Redox Signaling 19(9): 998-1011.

[71] Malea, P., Haritonidis, S., Stratis, I. (1994): Bioaccumulation of metals by Rhodophyta species at Antikyra Gulf (Greece) near an aluminium factory 1. - Botanica Marina 37: 505-513.

[72] Mao, Y., Chen, N., Cao, M., Chen, R., Guan, X., Wang, D. (2019): Functional characterization and evolutionary analysis of glycine-betaine biosynthesis pathway in red seaweed Pyropia yezoensis. - Marine Drugs 17(1): 70.

[73] McGlathery, K. J., Sundbäck, K., Anderson, I. C. (2007): Eutrophication in shallow coastal bays and lagoons: the role of plants in the coastal filter. - Marine Ecology Progress Series 348: 1-18.

[74] Mirshad, P. P., Puthur, J. T. (2016): Arbuscular mycorrhizal association enhances drought tolerance potential of promising bioenergy grass (Saccharum arundinaceum Retz.). Environmental Monitoring and Assessment 188(7): 425. 
[75] Monneveux, P., Nemmar, M. (1986): Contribution à l'étude de la résistance à la sécheresse chez le blé tendre (Triticum aestivum L.) et chez le blé dur (Triticum durum Desf.): étude de l'accumulation de la proline au cours du cycle de développement. Agronomie 6(6): 583-590.

[76] Moore, M. N., Depledge, M. H., Readman, J. W., Paul Leonard, D. R. (2004): An integrated biomarker-based strategy for ecotoxicological evaluation of risk in environmental management. - Mutation Research - Fundamental and Molecular Mechanisms of Mutagenesis 552(1-2): 247-268.

[77] Morin, S. (2006): Bioindication des effets des pollutions metalliques sur les communautes de diatomees benthiques approches in situ et experimentales. - PhD Thesis, Bordeaux University, France.

[78] Mouradi, A., Bennasser, L., Gloaguen, V., Mouradi, A., Zidane, H., Givernaud, T. (2014): Accumulation of heavy metals by macroalgae along the Atlantic coast of Morocco between El Jadida and Essaouira. - World Journal of Biological Research 6(1): 1-9.

[79] Mukherjee, S., Chakraborty, A., Mondal, S., Saha, S., Haque, A., Paul, S. (2019): Assessment of common plant parameters as biomarkers of air pollution. - Environmental Monitoring and Assessment 191(6): 400.

[80] Munda, I. M. (1980): Survey of the algal biomass in the polluted area around Rovinj (Istrian coast, North Adriatic). - Acta Adriatica 21: 333-354.

[81] Munda, I. M. (1982): The effects of organic pollution on the distribution of fucoid algae from the Istrian coast (vicinity of Rovinj). - Acta Adriatica 23: 329-337.

[82] Murray, S. N., Ambrose, R. F., Dethier, M. N. (2002): Methods for performing monitoring, impact, and ecological studies on rocky shores. - MMS OCS Study 70: 217.

[83] Mwambo, F. (2007): Land Evaluation in Essaouira Province - Morocco. - Ministry of Foreign Affairs, Istituto Agronomico per L'oltremare, Florence, Italy.

[84] Nannini, M., De Marchi, L., Lombardi, C., Ragazzola, F. (2015): Effects of thermal stress on the growth of an intertidal population of Ellisolandia elongata (Rhodophyta) from NW Mediterranean Sea. - Marine Environmental Research 112(Pt B): 11-19.

[85] Nassar, M. Z., El-Din, N. G. S., Gharib, S. M. (2015): Phytoplankton variability in relation to some environmental factors in the eastern coast of Suez Gulf, Egypt. Environmental Monitoring and Assessment 187(10): 648.

[86] Nixon, S. W. (1995): Coastal marine eutrophication: a definition, social causes, and future concerns. - Ophelia 41(1): 199-219.

[87] Noisette, F., Egilsdottir, H., Davoult, D., Martin, S. (2013): Physiological responses of three temperate coralline algae from contrasting habitats to near-future ocean acidification. - Journal of Experimental Marine Biology and Ecology 448: 179-187.

[88] Nomura, M., Muramoto, Y., Yasuda, S., Takabe, T., Kishitani, S. (1995): The accumulation of glycinebetaine during cold acclimation in early and late cultivars of barley. - Euphytica 83(3): 247-250.

[89] Orfanidis, S., Panayotidis, P., Ugland, K. I. (2011): Ecological Evaluation Index continuous formula (EEI-c) application: a step forward for functional groups, the formula and reference condition values. - Mediterranean Marine Science 12(1): 199-231.

[90] Pereira, P., de Pablo, H., Rosa-Santos, F., Pacheco, M., Vale, C. (2009): Metal accumulation and oxidative stress in Ulva sp. substantiated by response integration into a general stress index. - Aquatic Toxicology 91(4): 336-345.

[91] Reis, P. A., Cassiano, J., Veiga, P., Rubal, M., Sousa-Pinto, I. (2014): Fucus spiralis as monitoring tool of metal contamination in the northwest coast of Portugal under the European Water Framework Directives. - Environmental Monitoring and Assessment 186(9): 5447-5460.

[92] Rezzoum, N., Mouradi, A., Givernaud, T., Merzouk, N., Bennasser, L. (2016): Variations saisonnières des concentrations en quelques oligo-éléments et métaux lourds chez Fucus 
spiralis (Linnaeus, 1753) et Laminaria ochroleuca (Bachelot de la Pylaie, 1824) du littoral de la ville d'El Jadida, Maroc. - Afrique Science 12(3): 293-305.

[93] Riadi, H. (1998): Etude nationale sur la biodiversité, algues marines. - Direction de l'observation, des études et de la coordination. Observatoire Nationale de l'Environnement du Maroc O.N.E.M.

[94] Rodier, J. (2009): L'analyse de l'eau. 9ème édition. - Dunod, Paris.

[95] Roleda, M. Y., Marfaing, H., Desnica, N., Jónsdóttir, R., Skjermo, J., Rebours, C., Nitschke, U. (2019): Variations in polyphenol and heavy metal contents of wildharvested and cultivated seaweed bulk biomass: health risk assessment and implication for food applications. - Food Control 95: 121-134.

[96] Rubal, M., Veiga, P., Reis, P. A., Bertocci, I., Sousa-Pinto, I. (2014): Effects of subtle pollution at different levels of biological organisation on species-rich assemblages. Environmental Pollution 191: 101-110.

[97] Sabri, H., Cherifi, O., Maarouf, A., Cheggour, M., Bertrand, M., Mandi, L. (2017): Wastewater impact on macroalgae biodiversity in Essaouira coast (Morocco). - Journal of Materials and Environmental Science 8(3): 857-862.

[98] Saif, S., Khan, M. S. (2018): Assessment of toxic impact of metals on proline, antioxidant enzymes, and biological characteristics of Pseudomonas aeruginosa inoculated Cicer arietinum grown in chromium and nickel-stressed sandy clay loam soils. - Environmental Monitoring and Assessment 190(5): 290.

[99] Sava, D., Doroftei, E., Arcuş, M. (2011): Ecology and distribution of macrophytic red algae from the Romanian Black Sea coast. - Botanica Serbica 35(1): 37-41.

[100] Sawyer, C. N., McCarty, P. L. (1978): Chemistry for Environmental Engineering. McGraw Hill, New York.

[101] Scavia, D., Bricker, S. B. (2006): Coastal eutrophication assessment in the United States. - Biogeochemistry 79(1-2): 187-208.

[102] Schaffelke, B. (1999): Short-term nutrient pulses as tools to assess responses of coral reef macroalgae to enhanced nutrient availability. - Marine Ecology Progress Series 182: 305310.

[103] Schramm, W. (1999): Factors influencing seaweed responses to eutrophication: some results from EU-project EUMAC. - Journal of Applied Phycology 11(1): 69-78.

[104] Sfriso, A., Facca, C., La-Rocca, B., Ghetti, P. F. (2006): Set Up of Environmental Quality Indices Based on Seaweed Taxonomic Ratios for the Assessment of Transition Marine Areas: Venice, Lesina and Goro Study Cases. - In: APAT (ed.) Transition Waters Monitoring Research and Institutional Monitoring. Comparison Between Different Experiences. APAT, Venice, pp. 190-201 (in Italian).

[105] Sfriso, A., Facca, C., Ghetti, P. F. (2009): Validation of the Macrophyte Quality Index (MaQI) set up to assess the ecological status of Italian marine transitional environments. - Hydrobiologia 617(1): 117-141.

[106] Shams El-Din, N. G., Mohamedein, L. I., El-Moselhy, K. M. (2014): Seaweeds as bioindicators of heavy metals off a hot spot area on the Egyptian Mediterranean Coast during 2008-2010. - Environmental Monitoring and Assessment 186(9): 5865-5881.

[107] Si, J., Feng, Q., Yu, T., Zhao, C., Li, W. (2015): Variation in Populus euphratica foliar carbon isotope composition and osmotic solute for different groundwater depths in an arid region of China. - Environmental Monitoring and Assessment 187(11): 705.

[108] Silvia Taga, M., Miller, E. E., Pratt, D. E. (1984): Chia seeds as a source of natural lipid antioxidants. - Journal of the American Oil Chemists' Society 61(5): 928-931.

[109] Sivadas, S., Ingole, B., Nanajkar, M. (2010): Benthic polychaetes as good indicators of anthropogenic impact. - Indian Journal of Marine Sciences 39(2): 201-211.

[110] Steneck, R. S. (1986): The ecology of coralline algal crusts: convergent patterns and adaptative strategies. - Annual Review of Ecology and Systematics 17: 273-303. 


$$
-7738-
$$

[111] Topcuoglu, S., Guven, K. C., Balkis, N., Kirbasoglu, C. (2003): Heavy metal monitoring of marine algae from the Turkish Coast of the Black Sea, 1998-2000. - Chemosphere 52: 1683-1688.

[112] Varun, M., Jaggi, D., D’Souza, R., Paul, M. S., Kumar, B. (2015): Abutilon indicum L.: a prospective weed for phytoremediation. - Environmental Monitoring and Assessment 187(8): 527. 